Kragujevac Journal of Mathematics

Volume 44(2) (2020), Pages 205-216.

\title{
SOME COMMUTATIVITY THEOREMS FOR NEAR-RINGS WITH LEFT MULTIPLIERS
}

\author{
A. BOUA ${ }^{1}$, A. Y. ABDELWANIS ${ }^{2}$, AND A. CHILLALI $^{1}$
}

\begin{abstract}
Let $\mathcal{N}$ be a 3 -prime near-ring with the center $Z(\mathcal{N})$, and $U$ be a nonzero semigroup ideal of $\mathcal{N}$. In the present paper it is shown that a 3 -prime near-ring $\mathcal{N}$ is a commutative ring if and only if it admits left multipliers $\mathcal{F}$ and $G$ satisfying any one of the following properties: (i) $\mathcal{F}(x) G(y) \pm[x, y] \in Z(\mathcal{N})$; (ii) $\mathcal{F}(x) G(y) \pm x \circ y \in Z(\mathcal{N})$; (iii) $\mathcal{F}(x) G(y) \pm y x \in Z(\mathcal{N}) ;($ iv) $\mathcal{F}(x) G(y) \pm x y \in Z(\mathcal{N})$ and $(\mathrm{v}) \mathcal{F}([x, y]) \pm G(x \circ y) \in$ $Z(\mathcal{N})$ for all $x, y \in U$.
\end{abstract}

\section{INTRODUCTION}

In the present paper, $\mathcal{N}$ will denote a right near-ring with center $Z(\mathcal{N})$. A near-ring $\mathcal{N}$ is called zero-symmetric if $x 0=0$ for all $x \in \mathcal{N}$ (recall that right distributivity yields $0 x=0)$. A non empty subset $U$ of $\mathcal{N}$ is said to be a semigroup left (resp. right) ideal of $\mathcal{N}$ if $\mathcal{N} U \subseteq U$ (resp. $U \mathcal{N} \subseteq U$ ) and if $U$ is both a semigroup left ideal and a semigroup right ideal, it is called a semigroup ideal of $\mathcal{N}$. As usual for all $x, y$ in $\mathcal{N}$, the symbol $[x, y]$ stands for Lie product (commutator) $x y-y x$ and $x \circ y$ stands for Jordan product (anticommutator) $x y+y x$. We note that for a near-ring, $-(x+y)=-y-x$. Recall that $\mathcal{N}$ is 3-prime, that is, for all $a, b$ in $\mathcal{N}, a \mathcal{N} b=\{0\}$ implies that $a=0$ or $b=0 . \mathcal{N}$ is said to be 2-torsion free if whenever $2 x=0$, with $x \in \mathcal{N}$, then $x=0$. An additive mapping $d: \mathcal{N} \rightarrow \mathcal{N}$ is a derivation if $d(x y)=x d(y)+d(x) y$ for all $x, y \in \mathcal{N}$, or equivalently, as noted in [15], that $d(x y)=d(x) y+x d(y)$ for all $x, y \in \mathcal{N}$. The concept of derivation in rings has been generalized in several ways by various authors. Generalized derivation has been introduced already in rings by M. Brešar [7]. Also the notions of generalized derivation has been introduced in

Key words and phrases. 3-Prime near-ring, derivations, commutativity, generalized derivation, left multiplier.

2010 Mathematics Subject Classification. Primary: 16Y30. Secondary: 16N60, 16W25.

DOI $10.46793 / \mathrm{KgJMat} 2002.205 \mathrm{~B}$

Received: Decamber 18, 2017.

Accepted: March 30, 2018. 
near-rings by Öznur Gölbasi [11]. An additive mapping $\mathcal{F}: \mathcal{N} \rightarrow \mathcal{N}$ is called a right generalized derivation with associated derivation $d$ if $\mathcal{F}(x y)=\mathcal{F}(x) y+x d(y)$ for all $x, y \in \mathcal{N}$ and $\mathcal{F}$ is called a left generalized derivation with associated derivation $d$ if $\mathcal{F}(x y)=d(x) y+x \mathcal{F}(y)$ for all $x, y \in \mathcal{N}$. $\mathcal{F}$ is called a generalized derivation with associated derivation $d$ if it is both a left as well as a right generalized derivation with associated derivation $d$. An additive mapping $\mathcal{F}: \mathcal{N} \rightarrow \mathcal{N}$ is said to be a left (resp. right) multiplier (or centralizer) if $\mathcal{F}(x y)=\mathcal{F}(x) y$ (resp. $\mathcal{F}(x y)=x \mathcal{F}(y)$ ) holds for all $x, y \in \mathcal{N}$. $\mathcal{F}$ is said to be a multiplier if it is both left as well as right multiplier. Notice that a right (resp. left) generalized derivation with associated derivation $d=0$ is a left (resp. right) multiplier. Several authors investigated the commutativity in prime and semiprime rings admitting derivations and generalized derivations which satisfy appropriate algebraic conditions on suitable subset of the rings. For example, we refer the reader to $[1,2,4-6,8,10,12-14]$, where further references can be found. In $[2]$ the authors proved that the prime ring $\mathcal{R}$ must be commutative if $\mathcal{R}$ is equipped with a generalized derivation $F$ associated with a nonzero derivation $d$ satisfying any one of the following conditions:

(i) $\mathcal{F}(x) \mathcal{F}(y)-x y \in Z(\mathcal{R})$ for all $x, y \in I$;

(ii) $\mathcal{F}(x) \mathcal{F}(y)+x y \in Z(\mathcal{R})$ for all $x, y \in I$, where $I$ is a nonzero two sided ideal of $\mathcal{R}$.

From these identities, it is natural to consider the situations

(iii) $\mathcal{F}(x) \mathcal{F}(y)-y x \in Z(\mathcal{R})$ and

(iv) $\mathcal{F}(x) \mathcal{F}(y)+y x \in Z(\mathcal{R})$ for all $x, y$ in some suitable subset of $\mathcal{R}$, which is studied by Dhara et al. in [9].

Further, A. Ali et al. [10] proved that the prime ring $\mathcal{R}$ must be commutative if $\mathcal{R}$ is equipped with a generalized derivation $F$ associated with a nonzero derivation $d$ satisfying any one of the following conditions: $(i) \mathcal{F}(x) \mathcal{F}(y) \pm[x, y] \in Z(\mathcal{R})$ for all $x, y \in I,(i i) \mathcal{F}(x) \mathcal{F}(y) \pm x \circ y \in Z(\mathcal{R})$ for all $x, y \in I$. In this line of investigation, it is more interesting to study the identities in two directions replacing ring by near-ring and the generalized derivation by left multiplier. Motivated by the above results, here we continue this line of investigation by considering more general situations. More precisely, we explore the commutativity of a 3-prime ring provided with left multipliers $\mathcal{F}, G$ satisfying any one of the following identities:

(i) $\mathcal{F}(x) G(y) \pm[x, y] \in Z(\mathcal{N})$;

(ii) $\mathcal{F}(x) G(y) \pm x \circ y \in Z(\mathcal{N})$;

(iii) $\mathcal{F}(x) G(y) \pm y x \in Z(\mathcal{N})$;

(iv) $\mathcal{F}(x) G(y) \pm x y \in Z(\mathcal{N}) \in Z(\mathcal{N})$ and

(v) $\mathcal{F}([x, y]) \pm G(x \circ y) \in Z(\mathcal{N})$ for all $x, y \in \mathcal{N}$.

\section{Some Preliminaries}

In this section, we give some well known results of near-rings which will be used extensively in the forthcoming sections. 
Lemma 2.1. ([3, Lemma 1.3]). Let $\mathcal{N}$ be a 3-prime near-ring.

(i) If $z \in Z(\mathcal{N})-\{0\}$ and $x z \in Z(\mathcal{N})$, then $x \in Z(\mathcal{N})$.

(ii) If $U$ is a nonzero semigroup right ideal (resp. semigroup left ideal) and $x$ is an element of $\mathcal{N}$ such that $U x=\{0\}$ (resp. $x U=\{0\}$ ), then $x=0$.

Lemma 2.2. ([3, Lemma 1.5]). Let $\mathcal{N}$ be a 3-prime near-ring, such that $Z(\mathcal{N})$ contains a nonzero semigroup left ideal or semigroup right ideal. Then $\mathcal{N}$ is a commutative ring.

Lemma 2.3. ([3, Theorem 2.1]). Let $\mathcal{N}$ be a 3-prime near-ring, $U$ a nonzero semigroup left ideal or semigroup right ideal. If $\mathcal{N}$ admits a nonzero derivation $d$ such that $d(U) \subseteq Z(\mathcal{N})$, then $\mathcal{N}$ is a commutative ring.

Lemma 2.4. ([3, Lemma 1.4]). Let $\mathcal{N}$ be a 3-prime near-ring and $U$ a nonzero semigroup ideal of $\mathcal{N}$. If $x, y \in \mathcal{N}$ and $x U y=\{0\}$, then $x=0$ or $y=0$.

\section{Main Result}

Proposition 3.1. Let $\mathcal{N}$ be a 3-prime near-ring, and $U$ be a nonzero semigroup ideal of $\mathcal{N}$. If $\mathcal{N}$ admits nonzero left multiplier $\mathcal{F}$ and nonzero derivation $d$, then the following assertions are equivalent:

(i) $\mathcal{F}([x, y]) \in Z(\mathcal{N})$ for all $x, y \in U$;

(ii) $\mathcal{F}([d(x), y]) \in Z(\mathcal{N})$ for all $x, y \in U$;

(iii) $\mathcal{N}$ is a commutative ring.

Proof. It is obvious that (iii) implies (i) and (iii) implies (ii). So we need to prove that (i) $\Rightarrow$ (iii) and (ii) $\Rightarrow$ (iii).

(i) $\Rightarrow$ (iii) Suppose that

$$
\mathcal{F}([x, y]) \in Z(\mathcal{N}), \quad \text { for all } x, y \in U .
$$

Replacing $y$ by $y x$ in (3.1), we get

$$
(\mathcal{F}([x, y])) x \in Z(\mathcal{N}), \quad \text { for all } x, y \in U .
$$

Using Lemma 2.1 (i) together with (3.1), we obtain

$$
\mathcal{F}([x, y])=0 \text { or } x \in Z(\mathcal{N}), \quad \text { for all } x, y \in U .
$$

Which implies that

$$
\mathcal{F}([x, y])=0, \quad \text { for all } x, y \in U .
$$

Since $\mathcal{F}$ is left multiplier, (3.2) gives $\mathcal{F}(x) y=\mathcal{F}(y) x$ for all $x, y \in U$. Replacing $y$ by $[u, v] y$ and invoking (3.2), we get $\mathcal{F}(x)[u, v] y=0$ for all $u, v, x, y \in U$. Taking $x=x r$ where $r \in \mathcal{N}$ in the last expression, we arrive at $\mathcal{F}(x) \mathcal{N}[u, v] y=\{0\}$ for all $u, v, x, y \in U$. Using the 3 -primeness of $\mathcal{N}$ with the fact that $\mathcal{F} \neq 0$, we obtain $[u, v] U=\{0\}$ for all $u, v \in U$ and Lemma 2.1 (ii) gives $[u, v]=0$ for all $u, v \in U$ 
which forces that $\mathcal{N}$ is a commutative ring.

(ii) $\Rightarrow$ (iii) Assume that

$$
\mathcal{F}([d(x), y]) \in Z(\mathcal{N}), \quad \text { for all } x, y \in U .
$$

Substituting $y$ with $y d(x)$ in the pervious equation we obtain

$$
\mathcal{F}([d(x), y]) d(x) \in Z(\mathcal{N}), \quad \text { for all } x, y \in U .
$$

So by using Lemma 2.1(i) and (3.3), we get

$$
\mathcal{F}([d(x), y])=0 \text { or } d(x) \in Z(\mathcal{N}), \quad \text { for all } x, y \in U .
$$

Which implies that

$$
\mathcal{F}([d(x), y])=0, \quad \text { for all } x, y \in U .
$$

But $\mathcal{F}$ is left multiplier, then (3.5) implies that $\mathcal{F}(d(x)) y=\mathcal{F}(y) d(x)$ for all $x, y \in U$. Replacing $y$ by $[d(u), v] y$ and invoking (3.5), we get $\mathcal{F}(d(x))[d(u), v] y=0$ for all $u, v, x, y \in U$. Putting $x=x r$ where $r \in \mathcal{N}$ in the latter equation, we arrive at $\mathcal{F}(x) \mathcal{N}[d(u), v] y=\{0\}$ for all $u, v, x, y \in U$. By using the 3 -primeness of $\mathcal{N}$ and the fact that $\mathcal{F} \neq 0$, we get $[d(u), v] U=\{0\}$ for all $u, v \in U$. Hence, by Lemma 2.1 (ii) we obtain $[d(u), v]=0$ for all $u, v \in U$, which forces that $\mathcal{N}$ is a commutative ring by [4, Theorem 2.9].

It is clear that $i d_{\mathcal{N}}$ is a left multiplier of $\mathcal{N}$. If we replace $\mathcal{F}$ by $i d_{\mathcal{N}}$, we find a result similar to [4, Theorem 2.9] in the case of right near-rings.

Corollary 3.1. Let $\mathcal{N}$ be a 3-prime near-ring. If $U$ is a nonzero semigroup ideal of $\mathcal{N}$ and $d: \mathcal{N} \rightarrow \mathcal{N}$ be a derivation, then the following assertions are equivalent:

(i) $[x, y] \in Z(\mathcal{N})$ for all $x, y \in U$;

(ii) $[d(x), y] \in Z(\mathcal{N})$ for all $x, y \in U$;

(ii) $\mathcal{N}$ is a commutative ring.

Proposition 3.2. Let $\mathcal{N}$ be a 2-torsion free 3-prime near-ring, and $U$ be a nonzero semigroup ideal of $\mathcal{N}$. If $\mathcal{N}$ admits nonzero left multiplier $G$ and nonzero derivations $d$, then the following assertions are equivalent:

(i) $G(x \circ y) \in Z(\mathcal{N})$ for all $x, y \in U$;

(ii) $G(d(x) \circ y) \in Z(\mathcal{N})$ for all $x, y \in U$;

(iii) $\mathcal{N}$ is a commutative ring.

Proof. It is obvious that (iii) implies (i) and (iii) implies (ii). So it remains to prove that (i) $\Rightarrow$ (iii) and (ii) $\Rightarrow$ (iii).

(i) $\Rightarrow$ (ii) Suppose that

$$
G(x \circ y) \in Z(\mathcal{N}), \quad \text { for all } x, y \in U .
$$

Replacing $y$ by $y x$ in (3.6), we get

$$
G(x \circ y) x \in Z(\mathcal{N}), \quad \text { for all } x, y \in U .
$$


Using Lemma 2.1 (i) together with (3.6), we obtain

$$
G(x \circ y)=0 \text { or } x \in Z(\mathcal{N}), \quad \text { for all } x, y \in U .
$$

Suppose there exists $x_{0} \in U$ such that $G\left(x_{0} \circ y\right)=0$ for all $y \in U$. For $y=x_{0}$, we obtain $G\left(x_{0}\right) x_{0}=0$. Also, we have $G\left(x_{0}\right) y=-G(y) x_{0}$ for all $y \in U$. Putting $x_{0} y$ in place of $y$, we arrive at $G\left(x_{0}\right) U x_{0}=\{0\}$ and by Lemma 2.4, we get $G\left(x_{0}\right)=0$ or $x_{0}=0$ which implies that $G\left(x_{0}\right)=0$. In this case, our assumption gives $G(y) x_{0}=0$ for all $y \in U$. Replacing $y$ by $y t$, where $t \in N$, we get $G(y) N x_{0}=\{0\}$. By 3-primeness of $N$ with the fact that $G \neq 0$, we conclude that $x_{0}=0$. In this case, (3.7) implies that $U \subseteq Z(N)$ and Lemma 2.2 forces that $N$ is a commutative ring.

(ii) $\Rightarrow$ (iii) Assume that

$$
G(d(x) \circ y) \in Z(\mathcal{N}), \quad \text { for all } x, y \in U .
$$

Replacing $y$ by $y d(x)$ in (3.8), we obtain

$$
G(d(x) \circ y) d(x) \in Z(\mathcal{N}), \quad \text { for all } x, y \in U .
$$

Using Lemma 2.1 (i) together with (3.8), we obtain

$$
G(d(x) \circ y)=0 \text { or } d(x) \in Z(\mathcal{N}), \quad \text { for all } x, y \in U .
$$

Suppose there exists $x_{0} \in U$ such that $G\left(d\left(x_{0}\right) \circ y\right)=0$ for all $y \in U$. For $y=d\left(x_{0}\right)$, by 2 -torsion we get $G\left(d\left(x_{0}\right)\right) d\left(x_{0}\right)=0$. Also, we have $G\left(d\left(x_{0}\right)\right) y=-G(y) d\left(x_{0}\right)$ for all $y \in U$. Replacing $d\left(x_{0}\right) y$ in place of $y$, we arrive at $G\left(d\left(x_{0}\right)\right) U d\left(x_{0}\right)=\{0\}$ and by Lemma 2.4, we obtain $G\left(d\left(x_{0}\right)\right)=0$ or $d\left(x_{0}\right)=0$ which implies that $G\left(d\left(x_{0}\right)\right)=0$. Thus, our assumption gives $G(y) d\left(x_{0}\right)=0$ for all $y \in U$. Substituting $y$ by $y t$, where $t \in \mathcal{N}$, we get $G(y) \mathcal{N} d\left(x_{0}\right)=\{0\}$. By 3-primeness of $\mathcal{N}$ with the fact that $G \neq 0$, we conclude that $d\left(x_{0}\right)=0$. In this case, (3.9) implies that $d(U) \subseteq Z(\mathcal{N})$ and Lemma 2.3 forces that $\mathcal{N}$ is a commutative ring.

When $G=i d_{\mathfrak{N}}$, we find a result similar to [4, Theorem 2.10] in the case of right near-rings.

Corollary 3.2. Let $\mathcal{N}$ be a 2-torsion 3-prime near-ring. If $U$ is a nonzero semigroup ideal of $\mathcal{N}$ and $d: \mathcal{N} \rightarrow \mathcal{N}$ be a derivation, then the following assertions are equivalent:

(i) $x \circ y \in Z(\mathcal{N})$ for all $x, y \in U$;

(ii) $d(x) \circ y \in Z(\mathcal{N})$ for all $x, y \in U$;

(iii) $\mathcal{N}$ is a commutative ring.

Theorem 3.1. Let $\mathcal{N}$ be a 2-torsion free 3-prime near-ring, and $U$ be a nonzero semigroup ideal of $\mathcal{N}$. If $\mathcal{N}$ admits nonzero left multipliers $\mathcal{F}$ and $G$, then the following assertions are equivalent:

(i) $\mathcal{F}([x, y])+G(x \circ y) \in Z(\mathcal{N})$ for all $x, y \in U$;

(ii) $\mathcal{F}([x, y])-G(x \circ y) \in Z(\mathcal{N})$ for all $x, y \in U$;

(iii) $\mathcal{N}$ is a commutative ring. 
Proof. It is obvious that (iii) implies (i) and (iii) implies (ii). So we need to prove that (i) $\Rightarrow$ (iii) and (ii) $\Rightarrow$ (iii).

(i) $\Rightarrow$ (iii) Suppose that

$$
\mathcal{F}([x, y])+G(x \circ y) \in Z(\mathcal{N}), \quad \text { for all } x, y \in U .
$$

Replacing $y$ by $y x$ in (3.10), we get

$$
(\mathcal{F}([x, y])+G(x \circ y)) x \in Z(\mathcal{N}), \quad \text { for all } x, y \in U .
$$

Using Lemma 2.1(i) together with (3.10), we obtain

$$
\mathcal{F}([x, y])+G(x \circ y)=0 \text { or } x \in Z(\mathcal{N}), \quad \text { for all } x, y \in U .
$$

Suppose there exists $x_{0} \in Z(\mathcal{N})$, then (3.10) implies that $G(t+t) x_{0} \in Z(\mathcal{N})$ for all $t \in U$. By Lemma 2.1(i), we arrive at $x_{0}=0$ or $G(t+t) \in Z(\mathcal{N})$ for all $t \in U$, in this case, (3.11) becomes

$$
\mathcal{F}([x, y])+G(x \circ y)=0 \text { or } G(t+t) \in Z(\mathcal{N}), \quad \text { for all } x, y, t \in U .
$$

Assume that $\mathcal{F}([x, y])+G(x \circ y)=0$ for all $x, y \in U$. For $x=y$, we obtain $G\left(x^{2}\right)=G(x) x=0$ for all $x \in U$ and replacing $x$ by $x G(y)$ in our assumption, we have $\mathcal{F}([x G(y), y])+G(x G(y) \circ y)=0$ for all $x, y \in U$ and developing this equation, we find that $(G(y)-\mathcal{F}(y)) U G(y)=\{0\}$ for all $y \in U$. Using Lemma 2.4, we find that either $G(y)-\mathcal{F}(y)=0$ or $G(y)=0$ for all $y \in U$. Suppose there exists $y_{0} \in U$ such that $G\left(y_{0}\right)-\mathcal{F}\left(y_{0}\right)=0$ and replacing $y$ by $y_{0}$ in $\mathcal{F}([x, y])+G(x \circ y)=0$, we arrive at $(\mathcal{F}(x)+G(x)) y_{0}=0$ for all $x \in U$. Taking $x t$ in place of $x$, where $t \in \mathcal{N}$ the last expression becomes $(\mathcal{F}(x)+G(x)) \mathcal{N} y_{0}=\{0\}$ for all $x \in U$. By 3-primeness of $\mathcal{N}$, we get $\mathcal{F}=-G$ or $y_{0}=0$. Since $G \neq 0$, in all the cases, we obtain that $\mathcal{F}=-G$ which forces that $\mathcal{F}([x, y])=\mathcal{F}(x \circ y)$ for all $x, y \in U$ and developing this expression, we find that $\mathcal{F}(y) x=0$ for all $x, y \in U$. Using Lemma 2.1(ii), we conclude that $\mathcal{F}(U)=\{0\}$. Since $\mathcal{N} U \subseteq U$, then $\mathcal{F}(\mathcal{N}) U=\{0\}$. By Lemma 2.1(ii), we obtain $\mathcal{F}=0$, a contradiction.

Assume that $G(t+t) \in Z(\mathcal{N})$ for all $t \in U$. Putting $t=t r$, where $r \in \mathcal{N}$, then $G(t r+t r)=G(t+t) r \in Z(\mathcal{N})$ for all $t \in U, r \in \mathcal{N}$. Using Lemma 2.1(i), we obtain $G(t+t)=0$ or $r \in Z(\mathcal{N})$ for all $t \in U, r \in \mathcal{N}$ and using 2-torsion freeness of $\mathcal{N}$ together with $G \neq 0$, we conclude that $\mathcal{N}$ is a commutative ring.

The proof of (ii) $\Rightarrow$ (iii) is similar to (i) $\Rightarrow$ (iii).

In particular, when $G=i d_{\mathfrak{N}}$, then we have the following corollary.

Corollary 3.3. Let $\mathcal{N}$ be a 2-torsion free 3-prime near-ring, and $U$ be a nonzero semigroup ideal of $\mathcal{N}$. If $\mathcal{N}$ admits a nonzero left multiplier $\mathcal{F}$, then the following assertions are equivalent:

(i) $\mathcal{F}([x, y])+x \circ y \in Z(\mathcal{N})$ for all $x, y \in U$;

(ii) $\mathcal{F}([x, y])-x \circ y \in Z(\mathcal{N})$ for all $x, y \in U$;

(iii) $\mathcal{N}$ is a commutative ring.

In particular, when $\mathcal{F}$ is an identity map, then we have the following result. 
Corollary 3.4. Let $\mathcal{N}$ be a 2-torsion free 3-prime near-ring, and $U$ be a nonzero semigroup ideal of $\mathcal{N}$. If $\mathcal{N}$ admits a nonzero left multiplier $G$, then the following assertions are equivalent:

(i) $[x, y]+G(x \circ y) \in Z(\mathcal{N})$ for all $x, y \in U$;

(ii) $[x, y]-G(x \circ y) \in Z(\mathcal{N})$ for all $x, y \in U$;

(iii) $\mathcal{N}$ is a commutative ring.

In a ring $\mathcal{R}$, if $\mathcal{F}$ is a left multiplier, then $\mathcal{F} \pm i d_{\mathcal{R}}$ is also a left multiplier, where $i d_{\mathcal{R}}$ denotes the identity mapping on $\mathcal{R}$. By substituting $\mathcal{F} \pm i d_{\mathcal{R}}$ in place of $\mathcal{F}$ in Theorem 3.1 , we get the following result.

Corollary 3.5. Let $\mathcal{R}$ be a prime ring of characteristic not 2 , and $U$ be a nonzero ideal of $\mathcal{R}$. If $\mathcal{R}$ admits nonzero left multipliers $\mathcal{F}$ and $G$, then the following assertions are equivalent:

(i) $\mathcal{F}([x, y])+G(x \circ y) \pm[x, y] \in Z(\mathcal{R})$ for all $x, y \in U$;

(ii) $\mathcal{F}([x, y])-G(x \circ y) \pm[x, y] \in Z(\mathcal{R})$ for all $x, y \in U$;

(iii) $\mathcal{R}$ is commutative.

In particular, when $G$ is replaced by $G \pm i d_{\mathcal{R}}$, then we have the following.

Corollary 3.6. Let $\mathcal{R}$ be a prime ring of characteristic not 2 , and $U$ be a nonzero ideal of $\mathcal{R}$. If $\mathcal{R}$ admits nonzero left multipliers $\mathcal{F}$ and $G$, then the following assertions are equivalent:

(i) $\mathcal{F}([x, y])+G(x \circ y) \pm x \circ y \in Z(\mathcal{R})$ for all $x, y \in U$;

(ii) $\mathcal{F}([x, y])-G(x \circ y) \pm x \circ y \in Z(\mathcal{R})$ for all $x, y \in U$;

(iii) $\mathcal{R}$ is commutative.

In particular, when $\mathcal{F}$ and $G$ are replaced by $\mathcal{F} \pm i d_{\mathcal{R}}$ and $G \pm i d_{\mathcal{R}}$ respectively, then we have the following corollary.

Corollary 3.7. Let $\mathcal{R}$ be a prime ring of characteristic not 2 , and $U$ be a nonzero ideal of $\mathcal{R}$. If $\mathcal{R}$ admits nonzero left multipliers $\mathcal{F}$ and $G$, then the following assertions are equivalent:

(i) $\mathcal{F}([x, y])+G(x \circ y) \pm 2 x y \in Z(\mathcal{R})$ for all $x, y \in U$;

(ii) $\mathcal{F}([x, y])-G(x \circ y) \pm 2 y x \in Z(\mathcal{R})$ for all $x, y \in U$;

(iii) $\mathcal{R}$ is commutative.

Theorem 3.2. Let $\mathcal{N}$ be a 3-prime near-ring, and $U$ be a nonzero semigroup ideal of $\mathcal{N}$. If $\mathcal{N}$ admits left multipliers $\mathcal{F}$ and $G$, then the following assertions are equivalent:

(i) $\mathcal{F}(x) G(y)-[x, y] \in Z(\mathcal{N})$ for all $x, y \in U$;

(ii) $\mathcal{F}(x) G(y)-x \circ y \in Z(\mathcal{N})$ for all $x, y \in U$;

(iii) $\mathcal{N}$ is a commutative ring.

Proof. It is obvious that (iii) implies (i) and (iii) implies (ii). So we need to prove that (i) $\Rightarrow$ (iii) and (ii) $\Rightarrow$ (iii). 
(i) $\Rightarrow$ (iii) If $\mathcal{F}=0$ or $G=0$, then $-[x, y] \in Z(\mathcal{N})$ for all $x, y \in U$. Replacing $y$ by $y x$, we get $(-[x, y]) x \in Z(\mathcal{N})$ for all $x, y \in U$ and using Lemma 2.1(i), we obtain $[x, y]=0$ or $x \in Z(\mathcal{N})$ for all $x, y \in U$ which implies that $[x, y]=0$ for all $x, y \in U$. Using Corollary 3.1, we conclude that $\mathcal{N}$ is a commutative ring.

Suppose that $\mathcal{F} \neq 0$ and $G \neq 0$, we have

$$
\mathcal{F}(x) G(y)-[x, y] \in Z(\mathcal{N}), \quad \text { for all } x, y \in U .
$$

Replacing $y$ by $y x$ in (3.13) and using the fact that $[x, y x]=([x, y]) x$, we get

$$
(\mathcal{F}(x) G(y)-[x, y]) x \in Z(\mathcal{N}), \quad \text { for all } x, y \in U .
$$

By Lemma 2.1 (i) together with (3.13), we obtain

$$
\mathcal{F}(x) G(y)=[x, y] \text { or } x \in Z(\mathcal{N}), \quad \text { for all } x, y \in U \text {. }
$$

Suppose there exists $x_{0} \in U$ such that $\mathcal{F}\left(x_{0}\right) G(y)=\left[x_{0}, y\right]$ for all $y \in U$. Taking $y r$ instead of $y$, where $r \in \mathcal{N}$, we get $\mathcal{F}\left(x_{0}\right) G(y) r=\left[x_{0}, y r\right]$ for all $y \in U, r \in \mathcal{N}$ which implies that $\left[x_{0}, y\right] r=\left[x_{0}, y r\right]$ for all $y \in U, r \in \mathcal{N}$ and developing this expression, we arrive at $y\left[x_{0}, r\right]=0$ for all $y \in U, r \in \mathcal{N}$. Using Lemma 2.1 (ii), we obtain that $x_{0} \in Z(\mathcal{N})$ in this case, (3.2) becomes $x \in Z(\mathcal{N})$ for all $x \in U$ which forces that $\mathcal{N}$ is a commutative ring by Lemma 2.2 .

(ii) $\Rightarrow$ (iii) If $\mathcal{F}=0$ or $G=0$, then $-(x \circ y) \in Z(\mathcal{N})$ for all $x, y \in U$. Replacing $y$ by $y x$ we get $(-x \circ y) x \in Z(\mathcal{N})$ for all $x, y \in U$ and using Lemma 2.1 (i), we obtain $x \circ y=0$ or $x \in Z(\mathcal{N})$ for all $x, y \in U$. Using the same techniques as used in the proof of $[4$, Theorem 2.10], we conclude that $\mathcal{N}$ is a commutative ring.

Now assume that $\mathcal{F} \neq 0$ and $G \neq 0$, we have

$$
\mathcal{F}(x) G(y)-x \circ y \in Z(\mathcal{N}), \quad \text { for all } x, y \in U .
$$

Putting $y x$ instead of $y$ in (3.15) and using the fact that $x \circ y x=(x \circ y) x$, we get

$$
(\mathcal{F}(x) G(y)-x \circ y) x \in Z(\mathcal{N}), \quad \text { for all } x, y \in U .
$$

By Lemma 2.1(i) and using (3.15), we obtain

$$
\mathcal{F}(x) G(y)=x \circ y \text { or } x \in Z(\mathcal{N}), \quad \text { for all } x, y \in U .
$$

If there exists $x_{0} \in U$ such that $\mathcal{F}\left(x_{0}\right) G(y)=x_{0} \circ y$ for all $y \in U$. Taking $y r$ instead of $y$, where $r \in \mathcal{N}$, we obtain $\mathcal{F}\left(x_{0}\right) G(y) r=x_{0} \circ y r$ for all $y \in U, r \in \mathcal{N}$ this reduces to $\left(x_{0} \circ y\right) r=x_{0} \circ y r$ for all $y \in U, r \in \mathcal{N}$, so $y\left[x_{0}, r\right]=0$ for all $y \in U, r \in \mathcal{N}$. By Lemma 2.1 (ii), we conclude that $x_{0} \in Z(\mathcal{N})$, in this case, (3.16) becomes $x \in Z(\mathcal{N})$ for all $x \in U$. By Lemma 2.2, we conclude that $\mathcal{N}$ is a commutative ring.

Using similar techniques with necessary variations, we get the following Theorem. We skip the details of the proof just to avoid repetition.

Theorem 3.3. Let $\mathcal{N}$ be a 3-prime near-ring, and $U$ be a nonzero semigroup ideal of $\mathcal{N}$. If $\mathcal{N}$ admits left multipliers $\mathcal{F}$ and $G$, then the following assertions are equivalent:

(i) $\mathcal{F}(x) G(y)+[x, y] \in Z(\mathcal{N})$ for all $x, y \in U$;

(ii) $\mathcal{F}(x) G(y)+x \circ y \in Z(\mathcal{N})$ for all $x, y \in U$; 
(iii) $\mathcal{N}$ is a commutative ring.

If we put $F=G$ in Theorem 3.2 and Theorem 3.3, we obtain the following result.

Corollary 3.8. Let $\mathcal{N}$ be a 3-prime near-ring, and $U$ be a nonzero semigroup ideal of $\mathcal{N}$. If $\mathcal{N}$ admits left multiplier $\mathcal{F}$, then the following assertions are equivalent:

(i) $\mathcal{F}(x) \mathcal{F}(y) \pm[x, y] \in Z(\mathcal{N})$ for all $x, y \in U$;

(ii) $\mathcal{F}(x) \mathcal{F}(y) \pm x \circ y \in Z(\mathcal{N})$ for all $x, y \in U$;

(iii) $\mathcal{N}$ is a commutative ring.

Theorem 3.4. Let $\mathcal{N}$ be a 3-prime near-ring, and $U$ be a nonzero semigroup ideal of $\mathcal{N}$. If $\mathcal{N}$ admits left multipliers $\mathcal{F}$ and $G$, then the following assertions are equivalent:

(i) $\mathcal{F}(x) G(y)-y x \in Z(\mathcal{N})$ for all $x, y \in U$;

(ii) $\mathcal{F}(x) G(y)+y x \in Z(\mathcal{N})$ for all $x, y \in U$;

(iii) $\mathcal{N}$ is a commutative ring.

Proof. It is clear that (iii) implies (i) and (iii) implies (ii). It remains to show that (i) $\Rightarrow$ (iii) and (ii) $\Rightarrow$ (iii).

(i) $\Rightarrow$ (iii) If $\mathcal{F}=0$ or $G=0$, then $-y x \in Z(\mathcal{N})$ for all $x, y \in U$, so $-y x z \in Z(\mathcal{N})$ for all $x, y, z \in U$. Using Lemma 2.1 (i), we obtain $-y x=0$ or $z \in Z(\mathcal{N})$ for all $x, y, z \in U$. Using Lemma 2.1 (ii) and Lemma 2.2 with the fact that $U \neq\{0\}$, we conclude that $\mathcal{N}$ is a commutative ring.

Suppose that $\mathcal{F} \neq 0$ and $G \neq 0$, we have

$$
\mathcal{F}(x) G(y)-y x \in Z(\mathcal{N}), \text { for all } x, y \in U .
$$

Replacing $y$ by $y x$ in (3.17), we get

$$
(\mathcal{F}(x) G(y)-y x) x \in Z(\mathcal{N}), \quad \text { for all } x, y \in U .
$$

By Lemma 2.1 (i), the last expression becomes

$$
\mathcal{F}(x) G(y)=y x \text { or } x \in Z(\mathcal{N}), \text { for all } x, y \in U \text {. }
$$

Suppose there exists $x_{0} \in U$ such that $\mathcal{F}\left(x_{0}\right) G(y)=y x_{0}$ for all $y \in U$. Taking $y r$ instead of $y$, where $r \in \mathcal{N}$, we find that $\mathcal{F}\left(x_{0}\right) G(y) r=y r x_{0}$ for all $y \in U, r \in \mathcal{N}$ which implies that $y x_{0} r=y r x_{0}$ for all $y \in U, r \in \mathcal{N}$ and therefore $y\left[x_{0}, r\right]=0$ for all $y \in U$, $r \in \mathcal{N}$. Using Lemma 2.1 (ii), we obtain that $x_{0} \in Z(\mathcal{N})$ in this case, (3.18) becomes $x \in Z(\mathcal{N})$ for all $x \in U$ which forces that $\mathcal{N}$ is a commutative ring by Lemma 2.2.

(ii) $\Rightarrow$ (iii) Using the same tips that have used in the proof of (i) $\Rightarrow$ (iii), we get the desired result.

For $G=\mathcal{F}$, we have the following result.

Corollary 3.9. Let $\mathcal{N}$ be a 3-prime near-ring, and $U$ be a nonzero semigroup ideal of $\mathcal{N}$. If $\mathcal{N}$ admits a left multiplier $\mathcal{F}$, then the following assertions are equivalent:

(i) $\mathcal{F}(x) F(y)-y x \in Z(\mathcal{N})$ for all $x, y \in U$;

(ii) $\mathcal{F}(x) F(y)+y x \in Z(\mathcal{N})$ for all $x, y \in U$;

(iii) $\mathcal{N}$ is a commutative ring. 
Theorem 3.5. Let $\mathcal{N}$ be a 3-prime near-ring, and $U$ be a nonzero semigroup ideal of $\mathcal{N}$. If $\mathcal{N}$ admits left multipliers $\mathcal{F}$ and $G$ such that $\mathcal{F}(x) G(y)-x y \in Z(\mathcal{N})$ for all $x, y \in U$ or $\mathcal{F}(x) G(y)+x y \in Z(\mathcal{N})$ for all $x, y \in U$, then $\mathcal{N}$ is a commutative ring or $\mathcal{F}$ is a right multiplier.

Proof. If $\mathcal{F}=0$ or $G=0$, then $-x y \in Z(\mathcal{N})$ for all $x, y \in U$. Using the same proof that we have used in the beginning of Theorem 3.3, we obtain the required result. Now suppose that

$$
\mathcal{F}(x) G(y)-x y \in Z(\mathcal{N}), \quad \text { for all } x, y \in U .
$$

Putting $y z$ in place of $y$ in (3.19), we get

$$
(\mathcal{F}(x) G(y)-x y) z \in Z(\mathcal{N}), \quad \text { for all } x, y, z \in U .
$$

Using Lemma 2.1(i) and (3.19), the above expression implies that

$$
\mathcal{F}(x) G(y)=x y \text { or } z \in Z(\mathcal{N}), \quad \text { for all } x, y, z \in U
$$

By Lemma 2.2, we obtain

$$
\mathcal{F}(x) G(y)=x y, \quad \text { for all } x, y \in U \text { or } \mathcal{N} \text { is a commutative ring. }
$$

Assume that $\mathcal{F}(x) G(y)=x y$ for all $x, y \in U$. Taking $x u$ instead of $x$, we get $\mathcal{F}(x) u G(y)=x u y=x \mathcal{F}(u) G(y)$ for all $x, y, u \in U$, so $(\mathcal{F}(x) u-x \mathcal{F}(u) G(y)=0$ for all $x, y, u \in U$. Replacing $u$ by $u r$, where $r \in \mathcal{N}$, we arrive at $(\mathcal{F}(x) u-x \mathcal{F}(u)) \mathcal{N} G(y)=\{0\}$ for all $x, y, u \in U$. By 3-primeness of $\mathcal{N}$, we get either $G(y)=0$ or $\mathcal{F}(x) u=x \mathcal{F}(u)$ for all $x, y, u \in U$. If $G(y)=0$ for all $y \in U$, taking $r y$ instead of $y$, we find that $G(r) U=\{0\}$ for all $r \in \mathcal{N}$ and using Lemma 2.1(ii), we get $G=0$; a contradiction. If $\mathcal{F}(x) u=x \mathcal{F}(u)$ for all $x, u \in U$. Replacing $r x$ in place of $x$, we get $(\mathcal{F}(r) x-r \mathcal{F}(x)) U=$ $\{0\}$ for all $x \in U, r \in \mathcal{N}$. By Lemma 2.1(ii), we obtain $\mathcal{F}(r) x=r \mathcal{F}(x)$ for all $x \in U$, $r \in \mathcal{N}$. Taking $t x$ instead of $x$, where $t \in \mathcal{N}$ and using Lemma 2.1 (ii) again, we conclude that $\mathcal{F}(r) t=r \mathcal{F}(t)$ for all $r, t \in \mathcal{N}$ which forces that $\mathcal{F}$ is a right multiplier.

If $\mathcal{F}(x) G(y)+x y \in Z(\mathcal{N})$ for all $x, y \in U$, using the same techniques as have used in (i) $\Rightarrow$ (iii), we get the required result.

In particular, when $G=\mathcal{F}$, then we have the following.

Corollary 3.10. Let $\mathcal{N}$ be a 3-prime near-ring, and $U$ be a nonzero semigroup ideal of $\mathcal{N}$. If $\mathcal{N}$ admits a left multiplier $\mathcal{F}$ such that $\mathcal{F}(x) \mathcal{F}(y)-x y \in Z(\mathcal{N})$ for all $x, y \in U$ or $\mathcal{F}(x) \mathcal{F}(y)+x y \in Z(\mathcal{N})$ for all $x, y \in U$, then $\mathcal{N}$ is a commutative ring or $\mathcal{F}$ is a right multiplier.

The following example demonstrates that our results are not true for arbitrary near-rings.

Example 3.1. Suppose that $S$ is any right near-ring. Let

$$
\mathcal{N}=\left\{\left(\begin{array}{ccc}
0 & a & b \\
0 & 0 & c \\
0 & 0 & 0
\end{array}\right) \mid a, b, c \in S\right\} \text { and } U=\left\{\left(\begin{array}{ccc}
0 & 0 & u \\
0 & 0 & 0 \\
0 & 0 & 0
\end{array}\right) \mid u \in S\right\}
$$


Define maps $\mathcal{F}, G: \mathcal{N} \rightarrow \mathcal{N}$ such that

$$
\mathcal{F}\left(\begin{array}{ccc}
0 & a & b \\
0 & 0 & c \\
0 & 0 & 0
\end{array}\right)=\left(\begin{array}{ccc}
0 & 0 & a \\
0 & 0 & 0 \\
0 & 0 & 0
\end{array}\right) \text { and } G\left(\begin{array}{ccc}
0 & a & b \\
0 & 0 & c \\
0 & 0 & 0
\end{array}\right)=\left(\begin{array}{ccc}
0 & 0 & 0 \\
0 & 0 & c \\
0 & 0 & 0
\end{array}\right) \text {. }
$$

Then, it is easy to see that $\mathcal{N}$ is a right near-ring and $\mathcal{F}, G$ are left multipliers on $\mathcal{N}$ satisfying the following properties:

(i) $\mathcal{F}(x) G(y) \pm[x, y] \in Z(\mathcal{N})$;

(ii) $\mathcal{F}(x) G(y) \pm x \circ y \in Z(\mathcal{N})$;

(iii) $\mathcal{F}(x) G(y) \pm x y \in Z(\mathcal{N})$;

(iv) $\mathcal{F}(x) G(y) \pm y x \in Z(\mathcal{N})$;

(v) $\mathcal{F}([x, y]) \pm G(x \circ y) \in Z(\mathcal{N})$;

for all $x, y \in U$. However, $\mathcal{N}$ is not commutative.

The following example shows that the condition "F $([x, y]) \pm G(x \circ y) \in Z(\mathcal{N})$ for

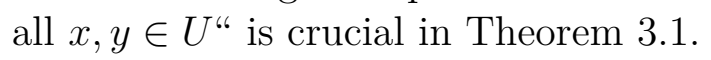

Example 3.2. Let $\mathcal{N}=M_{2}(\mathbb{Z})$ be the $2 \times 2$ matrix ring over $\mathbb{Z}$ and $\mathcal{F}, G: \mathcal{N} \rightarrow \mathcal{N}$ such that

$$
\mathcal{F}\left(\begin{array}{ll}
a & b \\
c & d
\end{array}\right)=\left(\begin{array}{ll}
a & b \\
0 & 0
\end{array}\right), \quad \text { for all } a, b, c, d \in \mathbb{Z} \text { and } G=\mathcal{F}
$$

It is easy to verify that $\mathcal{N}$ is a non-commutative prime ring which is 2-torsion free and $\mathcal{F}, G$ are left multipliers of $\mathcal{N}$. Moreover, for $A=\left(\begin{array}{ll}0 & 1 \\ 1 & 1\end{array}\right), B=\left(\begin{array}{ll}1 & 0 \\ 1 & 0\end{array}\right)$, we have $\mathcal{F}([A, B]) \pm G(A \circ B) \notin Z(\mathcal{N})$.

The following example demonstrate that the existence of "2-torsion free" in the hypotheses of Theorem 3.1 is essential.

Example 3.3. Let $\mathcal{N}=M_{2}\left(\mathbb{Z}_{2}\right)$ be the $2 \times 2$ matrix ring over the field $\mathbb{Z}_{2}$ and $\mathcal{F}=I d_{\mathcal{N}}$. It is easy to see that $\mathcal{N}$ is a non-commutative prime ring which is not 2-torsion free. Moreover, $\mathcal{N}$ satisfies the condition $\mathcal{F}([x, y]) \pm G(x \circ y) \in Z(\mathcal{N})$ for all $x, y \in \mathcal{N}$.

\section{REFERENCES}

[1] M. Ashraf and N. Rehman, On derivations and commutativity in prime rings, East-West J. Math. 3(1) (2001), 87-91.

[2] M. Ashraf, A. Ali and S. Ali, Some commutativity theorems for rings with generalized derivations, Southeast Asian Bull. Math. 31 (2007), 415-421.

[3] H. E. Bell, On derivations in near-rings II, in: Nearrings, Nearfields and K-Loops, (Hamburg, 1995), Math. Appl. 426, Kluwer Academic Publishers, Dordrecht, 1997.

[4] H. E. Bell, A. Boua and L. Oukhtite, Semigroup ideals and commutativity in 3-prime near rings, Comm. Algebra 43 (2015), 1757-1770.

[5] A. Boua, L. Oukhtite and H. E. Bell, Differential identities on semigroup ideals of right nearrings, Asian-Eur. J. Math. 6(4) (2013), Paper ID 1350050, 8 pages.

[6] A. Boua and L. Taoufiq, Some algebraic results involving derivations in 3-prime near-rings, Indian J. Math. 59(2) (2017), 147-160. 
[7] M. Brešar, On the distance of composition of two derivations to the generalized derivations, Glasg. Math. J. 33 (1991), 89-93.

[8] M. N. Daif and H. E. Bell, Remarks on derivations on semiprime rings, Int. J. Math. Math. Sci. 15 (1) (1992), 205-206.

[9] B. Dhara and S. Ali, On multiplicative (generalized)-derivations in prime and semiprime rings, Aequationes Math. 86(1-2) (2013), 65-79.

[10] A. Ali, B. Dhara, S. Khan and F. Ali, Multiplicative (generalized)-derivations and left ideals in semiprime rings, Hacet. J. Math. Stat. 44(6) (2015), 1293-1306

[11] Ö. Gölbasi, Notes on prime near-rings with generalized derivation, Southeast Asian Bull. Math. 30 (2006), 49-54.

[12] M. Hongan, A note on semiprime rings with derivation, Int. J. Math. Math. Sci. 20(2) (1997), 413-415.

[13] M. A. Quadri, M. S. Khan and N. Rehman, Generalized derivations and commutativity of prime rings, Indian J. Pure Appl. Math. 34(9) (2003), 1393-1396.

[14] N. Rehman, On commutativity of rings with generalized derivations, Math. J. Okayama Univ. 44 (2002), 43-49.

[15] X. K. Wang, Derivations in prime near-rings, Proc. Amer. Math. Soc. 121 (1994), 361-366

${ }^{1}$ Sidi Mohammed Ben Abdellah University,

POLYDiSCIPLINARY FACULTY, LSI,

TAZA, MOROCCO

Email address: abdelkarimboua@yahoo.fr

Email address: abdelhakim.chillali@usmba.ac.ma

${ }^{2}$ Department of Mathematics,

Faculty of Science, Cairo University,

GizA 12613, EGYPT

Email address: ayunis@sci.cu.edu.eg 